\title{
Análisis del proceso de transcripción en la expresión escrita de alumnos sordos
}

\section{Analysis of the transcription process in the writing of deaf students}

\author{
Rafaela GUTIÉRREZ CÁCERES \\ Universidad de Almería
}

Recibido: Enero 2012

Aceptado: Marzo 2012

\section{Resumen}

El presente trabajo analiza las competencias que poseen los alumnos sordos de Educación Secundaria en relación con el conocimiento y uso de la transcripción en la composición escrita. En este estudio cualitativo-individual, de carácter exploratorio, se ha realizado una "entrevista cognitiva", después de la elaboración de una redacción escrita espontánea de un texto narrativo. Posteriormente se ha aplicado el análisis de contenido de las entrevistas y de los textos escritos de acuerdo a un sistema de clasificación de categorías que ha sido validado con el procedimiento del "juicio de expertos". En el análisis de resultados se ha encontrado que, en términos generales, los alumnos sordos no poseen las habilidades necesarias para expresar por escrito las ideas, utilizando correctamente la estructura y la forma del texto. En cualquier caso, es preciso señalar la variabilidad que existe entre el alumnado según sus características individuales y contextuales.

Palabras clave: Proceso de transcripción, Escritura, Discapacidad auditiva, Educación Secundaria.

\begin{abstract}
This paper analyzes the skills that they have deaf students of secondary education in the knowledge and use of the cognitive transcription process. For data collection, has carried out a "cognitive interview", after spontaneous writing an essay of narrative text. Subsequently has applied the content analysis of interviews and written texts according to a categories ranking system that has been validated with the procedure of "experts' judgment". In the analysis of results has been found that, in general, deaf students of secondary education do not have the skills needed to express ideas in writing properly, using correct language structure and form of the text. In any case, it should be noted that there is variability among deaf students depending on their individual and contextual characteristics.
\end{abstract}

Keywords: Transcription Process, Writing, Deaf Students, Secondary Education.

Las personas sordas, en general, no son lectores ni escritores competentes. Tienen grandes dificultades para comprender textos escritos y escribir cuentos, resúmenes, 
cartas, exámenes,... Esto repercute en que sean escasas las personas sordas que superen la Educación Secundaria, logren acceder a estudios superiores y tengan un nivel de integración socio-laboral satisfactorio (Fernández Viader y Pertusa, 1996; Watson, 1999; Jáudenes, 2009).

Por otra parte, diversas investigaciones, tanto en el ámbito nacional como internacional han intentado dar respuesta a las dificultades que se plantean en la lectura de los niños sordos (Carrillo y Domínguez, 2010). En cambio, en el ámbito nacional las investigaciones sobre la composición escrita de personas sordas son insuficientes (Ramspott, 1991; Cambra, 1993, 1994; Pertusa y Fernández Viader, 1999; Gutiérrez Cáceres, 2004b, 2005b, 2005c, 2005d; Gutiérrez Cáceres y Salvador Mata, 2006, 2010). Por otro lado, los resultados de los estudios realizados, en su mayoría, en inglés, no son aplicables ni, por tanto, generalizables a otros idiomas, como en este caso, el español (Lacerte, 1989; Pace, Pontecorvo, Skliar y Volterra, 1994; Tur-Kaspa y Dromi, 1998; Teruggi, 2001, 2003). La evidencia de esta situación ha dado argumentos para focalizar el trabajo de investigación en la escritura de personas con discapacidad auditiva.

En concreto, en este artículo se presenta un estudio de casos sobre el proceso de transcripción en la expresión escrita de alumnos sordos de Educación Secundaria. Se trata de un estudio cualitativo-individual, de carácter exploratorio, el cual puede constituir una de las herramientas básicas que deben utilizar los profesionales de la educación en la toma de decisiones en situaciones concretas de actuación. En efecto, no se intenta diseñar una propuesta de intervención educativa de la expresión escrita de alumnos sordos, sino que se pretende realizar una evaluación curricular de las capacidades y las dificultades con el fin de fundamentar y adecuar la práctica educativa a las necesidades educativas que de aquélla se derivan. Asimismo, en este artículo se pretende obtener información útil que permite profundizar en el conocimiento teórico sobre la expresión escrita de las personas sordas.

En cuanto al concepto de "expresión escrita" y su significado, es necesario destacar que se encuentra muy relacionado con los enfoques de investigación que han ido surgiendo a lo largo del tiempo. Tradicionalmente, la escritura se definía como la representación de las palabras o del pensamiento con letras o signos, trazados con un instrumento en una determinada superficie (Holbrook, 1989; Zesiger, Deonna y Mayor, 2000).

Desde la década de los 70, siguiendo la orientación piagetiana (Sinclair, 1967, Ferreiro, 1979) y bajo la influencia de las ideas aportadas por Vigotsky $(1977,1978)$, la composición escrita se considera como una estructuración del pensamiento o una representación mental del sujeto desde un punto de vista cognitivo, así como también desde una perspectiva funcional y contextual definida como un instrumento básico de comunicación que se desarrolla en situaciones sociales concretas.

En este sentido, la expresión escrita es una habilidad cognitiva, que requiere no sólo la intervención de procesos cognitivos de bajo nivel, implicados en la traducción físico-motora de signos convencionales, sino también la coordinación de procesos cognitivos de alto nivel, necesarios para escribir adecuadamente un texto significativo 
y funcional en una determinada situación de comunicación (Flower y Hayes, 1981, 1984; Scardamalia y Bereiter, 1986; Hayes, 1996).

Así pues, adoptando la perspectiva de la Gramática del Texto, un texto no se refiere a una suma de unidades lingüísticas (palabras, oraciones,...), desconectadas entre sí, sino a una unidad lingüística comunicativa con entidad propia que posee una finalidad comunicativa concreta (comunicar mensajes significativos), se produce en una situación social específica y cuya estructura interna está regida por los principios de la gramática oracional (fonética, ortografía, morfosintaxis, léxico), y los del nivel profundo-interno de la organización textual (cohesión y coherencia) (Halliday y Hasan, 1976; Van Dijk, 1983; Bernárdez, 1987).

En definitiva, la expresión escrita se refiere a una competencia comunicativa, en la que se distinguen dos componentes:

- El producto o resultado. Es el componente cognitivo-formal, referido al "saber", es decir, a la representación mental por parte de la persona de la lengua escrita como sistema.

- El proceso o componente contextual-simbólico, que se refiere al "saber hacer", es decir, al uso que la persona hace de la lengua escrita en contextos sociales y significativos.

Ambos componentes se encuentran estrechamente relacionados entre sí, de tal manera que el producto de la expresión escrita está determinado por el proceso que realiza el sujeto. A su vez, la producción de textos escritos está influida por el resultado del proceso.

Dentro de este marco teórico, esta investigación se centró en el análisis de la transcripción desde la perspectiva del proceso y del producto. La transcripción es un proceso mental que hace referencia a las habilidades que la persona tiene para desarrollar por escrito el contenido, la estructura y la forma del texto (Flower y Hayes, 1981; Berninger y Whitaker, 1993; Berninger y otros, 1996; García Sánchez, 2002). Es decir, el proceso de transcripción hace referencia a un "conjunto de procesos implicados en la traslación de ideas, que están organizadas espacial o jerárquicamente, a una secuencia lineal, el texto, que puede ser leído linealmente" (Salvador Mata, 1997, 32). En la transcripción la persona se centra en los procesos cognitivos de bajo nivel (sintácticos, léxicos, grafo-motores,...), sin desviarse de la representación mental de ideas relacionadas con la escritura. Asimismo, en esta fase se pone en funcionamiento el conocimiento lingüístico, tanto de las convenciones (ortografía, grafía, puntuación,...) como de las reglas gramaticales (léxico y morfosintaxis) y de la cohesión discursiva y/o estructura textual (Salvador Mata, 2005).

A su vez, este proceso incluye varios sub-procesos cognitivos:

- Adecuación de las palabras a las ideas: habilidad para escribir las ideas atendiendo a la coherencia entre "lo escrito" y "lo pensado".

- Riqueza de vocabulario: conocimiento y uso de la dimensión léxica del lenguaje escrito. 
- Selección léxica: habilidad para estructurar las ideas por escrito centrándose en la elección de palabras adecuadas.

- Ordenación sintáctica: conocimiento y uso de la construcción sintáctica del lenguaje escrito.

En este campo existe mucha investigación con niños oyentes, pero relativamente poca se ha llevado a cabo con niños sordos. A partir de la revisión de algunas investigaciones recientes más representativas, abordadas desde diferentes perspectivas teóricas y metodológicas fundamentalmente centradas en el producto, se ha encontrado que en los textos escritos por sujetos sordos existen oraciones cortas y simples, por un lado, y por otro, surgen los siguientes errores sintácticos más frecuentes: ausencia o utilización innecesaria de preposiciones, ausencia de determinantes, de verbos y de nexos; uso inapropiado del tiempo verbal,... (Lacerte, 1989; Nadeau, Dubuisson y Gélinas-Chebat, 1991; Boisclair y Sirios, 1996; Tur-Kaspa y Dromi, 1998; Gutiérrez Cáceres, 2002a, 2002b, 2004a). No obstante, para el desarrollo adecuado de la competencia comunicativa en el alumnado sordo se requiere el análisis de las estrategias cognitivas de la composición relacionadas con el proceso de transcripción desde un enfoque basado en el discurso, como es en esta investigación. En este sentido, en este trabajo se ha planteado determinar cuáles son las necesidades educativas que tienen los alumnos sordos en la composición escrita y, en concreto, en el conocimiento y uso de la transcripción.

\section{Material y métodos}

El objetivo principal de la investigación ha sido evaluar las competencias de los alumnos sordos de Educación Secundaria en el proceso de transcripción de la expresión escrita. En concreto, se han planteado los siguientes interrogantes:

- ¿LLos alumnos sordos realizan y/o conocen el proceso de transcripción del texto?

- ¿Qué operaciones realizan al transcribir el texto y cómo realizan?

- ¿LLos alumnos sordos conocen las reglas sintácticas del texto escrito?

- ¿En qué aspectos sintácticos de los textos escritos encuentran dificultades?

De acuerdo con los objetivos específicos de la investigación, se ha adoptado una metodología descriptiva de carácter cualitativo, utilizando la estrategia metodológica del "estudio de casos".

Para ello, se ha contado con la participación de 10 alumnos, cuya edad está comprendida entre los 16 y los 20 años, salvo una alumna de 13. Seis cursan la enseñanza secundaria no obligatoria (bachillerato) y el resto la enseñanza secundaria obligatoria (ESO). La mayoría se encuentran escolarizados en un Instituto de Integración, salvo una alumna que procede de un Colegio Específico de Sordos (véase Cuadro 1). 
Atendiendo a las evaluaciones audiológicas, la mitad de los alumnos tiene un déficit auditivo de grado profundo y el resto tiene una pérdida auditiva severa, excepto un alumno que tiene una deficiencia auditiva de grado medio. Según el momento de aparición de la sordera, los alumnos son prelocutivos, es decir, se quedaron sordos antes de los cuatro años de edad aproximadamente, excepto una alumna, cuya situación en relación con esta característica es desconocida.

Con respecto al tipo de prótesis auditivas, la mayoría llevan audífonos. Sólo a dos alumnas se les ha colocado un implante coclear. Excepto tres sujetos, que utilizan sus audífonos de forma irregular, los demás emplean las prótesis auditivas con regularidad, haciendo un uso funcional de las mismas, lo cual incide en el aprendizaje y desarrollo del lenguaje oral.

\begin{tabular}{|c|c|c|c|c|c|c|c|c|c|c|}
\hline Alumno & Edad & Sexo & $\begin{array}{c}\text { Nivel } \\
\text { Instructivo }\end{array}$ & $\begin{array}{c}\text { Etapa } \\
\text { Educativa }\end{array}$ & $\begin{array}{l}\text { Modalidad } \\
\text { Educativa }\end{array}$ & $\begin{array}{c}\text { Grado } \\
\text { Déficit } \\
\text { Auditivo }\end{array}$ & $\begin{array}{c}\text { Momento } \\
\text { Déficit } \\
\text { Auditivo }\end{array}$ & $\begin{array}{c}\text { Tipo } \\
\text { Ayudas } \\
\text { Técnicas }\end{array}$ & $\begin{array}{c}\text { Uso } \\
\text { Ayudas } \\
\text { Técnicas }\end{array}$ & Padres \\
\hline 1 & 17 & $\mathrm{M}$ & $4^{\circ}$ & ESO & Específico & Profundo & & Audífonos & A veces & Oyentes \\
\hline 2 & 20 & $\mathrm{H}$ & $2^{\circ}$ & Bachiller. & Integración & Severo & Prelocutivo & Audífonos & Siempre & Sordos \\
\hline 4 & 19 & $\mathrm{H}$ & $2^{\circ}$ & Bachiller. & Integración & Severo & Prelocutivo & Audífonos & A veces & Sordos \\
\hline 5 & 17 & $\mathrm{H}$ & $1^{\circ} / 2^{\circ}$ & Bachiller. & Integración & Moderado & Prelocutivo & Audífonos & Siempre & Oyentes \\
\hline 6 & 17 & $\mathrm{H}$ & $1^{\circ} / 2^{\circ}$ & Bachiller. & Integración & Severo & Prelocutivo & Audífonos & Siempre & Oyentes \\
\hline 7 & 17 & $\mathrm{M}$ & $1^{\circ}$ & Bachiller. & Integración & Profundo & Prelocutivo & Implante C. & Siempre & Oyentes \\
\hline 10 & 16 & M & $3^{\circ}$ & ESO & Integración & Severo & Prelocutivo & Audífonos & A veces & Oyentes \\
\hline
\end{tabular}

Cuadro 1: Características de los alumnos

En el ámbito educativo formal, el modo de comunicación prioritario es el lenguaje oral. No obstante, para favorecer una adecuada recepción y comprensión de los mensajes orales, los profesores utilizan otros recursos adicionales, en función de las necesidades concretas de cada alumno, como la lectura labial, la escritura de palabras y de frases, la dactilología y signos que acompañan a la lengua oral y, sobre todo, el intérprete de Lengua de Signos Española y los servicios logopédicos.

Con respecto al tipo de lenguaje que se emplea en el ámbito familiar, la mayoría de los alumnos sordos, que son hijos de padres oyentes, no conocen o no utilizan la lengua de signos como lo hacen otros dos alumnos, hijos de padres sordos. No obstante, cuando acceden al centro educativo, tienen muchas posibilidades de aprender la lengua de signos, al comunicarse con sus compañeros sordos por este medio. 
Para la obtención de datos, se ha realizado una "entrevista cognitiva" después de que el alumno elaborara una redacción escrita espontánea de un texto. Así pues, primero se trataba de pedir a los alumnos sordos que expresaran por escrito, de forma individual y espontánea, un tema de carácter narrativo, relacionado básicamente con una historia, una película, un cuento,... Posteriormente, partiendo de un cuestionarioguía (Salvador Mata, 2000, 66-71), el entrevistador preguntaba al alumno sobre su percepción de los procesos cognitivos implicados en la expresión escrita, de forma que el entrevistado pudiera pensar, reflexionar y expresar abiertamente sus ideas.

La entrevista se ha realizado en sesiones individuales con cada uno de los alumnos. En el desarrollo de las sesiones se han considerado las necesidades concretas que podían tener los alumnos sordos en la comunicación social. Por ello, ha sido imprescindible la colaboración del intérprete de lengua de signos. En estas sesiones se ha empleado una cámara de vídeo para grabar las entrevistas, para su posterior transcripción y análisis.

En el análisis de datos se ha utilizado la técnica de "análisis de contenido" para clasificar, codificar y analizar el contenido de las entrevistas de acuerdo con el sistema de categorías, similar al empleado en otras investigaciones (Salvador Mata y García Guzmán, 2005). Las categorías se corresponden con las diferentes operaciones que están relacionadas con el proceso de transcripción. El sistema de categorías y el cuestionario-guía han sido validados con el procedimiento del "juicio de expertos". Asimismo, los análisis de las entrevistas han sido sometidos para su fiabilidad a la "triangulación de codificadores independientes".

De otra parte, el análisis de los textos escritos se ha realizado en función de un modelo diseñado por Salvador Mata $(1984,1999)$, a partir de las aportaciones de otros autores. El procedimiento de análisis obedece concretamente a unas reglas de delimitación y categorización de las disfunciones sintácticas, en función de unos criterios sintácticos-formales y semánticos. El modelo de análisis, al mismo tiempo que sirvió de guía en el desarrollo de la investigación, permitió incorporar nuevos elementos de estudio, en función de las características concretas y específicas de los textos escritos por alumnos sordos.

\section{Resultados: análisis e interpretación}

Los resultados obtenidos en el análisis e interpretación de las entrevistas permiten conocer en profundidad las distintas operaciones cognitivas que tienen lugar en el proceso de transcripción de la expresión escrita de alumnos sordos de Educación Secundaria.

En general, los alumnos sordos, antes de escribir, primero piensan las ideas y después escriben realizando, para ello, una selección léxica según su relación con el tema del texto: "Pues al principio cuando me pongo a escribir, antes lo pienso, ¿qué es lo que quiero expresar?, ¿qué es lo que quiero decir?. Depende de la situación, ¿qué es lo que he hecho antes?. Después empiezo a escribir, pero buscando también palabras, palabras en el diccionario que estén mejor escritas, depende de los 
sentimientos que quiera expresar" (sujeto 2).

Sólo un alumno va más allá, cuando afirma que utiliza una variedad de palabras para expresar por escrito sus ideas de forma adecuada: “... yo elijo las palabras que están relacionadas con lo que voy a escribir, también por ejemplo para expresarme, pues la forma, por si voy a hablar de un sentimiento dulce o si es un sentimiento así fuerte, también utilizo recursos literarios, metáforas, cosas relacionadas y cosas que son palabras que no valen para ese contexto, son palabras que no se utilizan para eso" (sujeto 2).

En todo caso, la mayoría señalan la importancia de realizar una adecuada selección léxica, ya que, en efecto, existen unas palabras que explican mejor que otras según el contexto comunicativo: "Es que no sé, es que depende un poco, vamos, en una carta, por ejemplo, va a dar igual, porque tú ahí tienes una confianza y es diferente. A ver, tú en clase, en un aula, te hace falta buscar una serie de palabras, como que sea más ... más culta, ¿no? más cultas esas palabras, es lo que te hace falta buscar otras palabras, si"' (sujeto 7).

No obstante, aunque piensen que sí hay otras palabras que expresan mejor lo que quieren escribir en un texto o redacción, los alumnos en general son conscientes de sus dificultades y dudas en cuanto al léxico del lenguaje escrito, ya que no encuentran las palabras adecuadas para expresar lo que querían decir:

- "Es que cuando hablo lo sé y cuando escribo ya no sé qué ponerle” (sujeto 3).

- “... No sé, pero creo que... que lo he escrito yo no son mejores que las... que otras palabras que pueden haber... no sé” (sujeto 10).

En efecto, no poseen las habilidades necesarias para expresar por escrito las ideas de forma adecuada, utilizando correctamente las palabras:

- "Sí, por ejemplo si esta palabra, yo he pensado, y el profesor dice que tiene otro sentido. Depende yo pongo esta palabra, yo pensaba este sentido y luego ése no es" (sujeto 3).

- "No sé, porque a mí me cuesta trabajo expresarme y después escribirlo. Pues no creo que encuentre todas las palabras claves..." (sujeto 10).

Y ante estas dificultades, recurren a diversas estrategias de apoyo para poder utilizar adecuadamente la selección léxica en la expresión escrita:

a) Consultar fuentes escritas de información:

“... las palabras raras pues las quito, las elimino, las dejo fuera, las palabras fáciles o por ejemplo algunos recuerdos si tú sabes, algunos nombres, entonces los busco en el diccionario si no los tengo claros ¿no?, o también los busco en el ordenador en Internet" (sujeto 1).

- "Por ejemplo, busca una palabra, pues la puedo buscar en el diccionario para cambiar otra palabras, para no repetir las mismas palabras. Entonces las cambio un poco. Así se puede aprender más vocabulario, más comprensión del texto" 
(sujeto 6).

- "Sí, muchas veces hay una palabra que tiene un significado y al final no es, pues tengo que buscar en el diccionario, antes de escribirlas, busco en el diccionario, a ver lo que significa, a ver si es un sinónimo o si no lo es, si es un sinónimo pues sí $y$ de todas formas busco el significado de la palabra para poder utilizarla bien" (sujeto 2).

b) Preguntar a otras personas:

- “... aprovecho también y pregunto al logopeda, ¿esta palabra qué significa?. Entonces voy aprovechando eso para luego plasmarlo mejor" (sujeto 2).

- "Para escribir una palabra... pues le pregunto a mi madre cuando no sé una palabra ...Luego mando la carta y mi amiga la lee bien..." (sujeto 8).

- "Le digo al profesor o mi madre... le pregunto" (sujeto 9).

c) Otras:

- "Sí, me pongo a copiar la palabra o si puedo la busco en el diccionario, depende, si no lo manejas no puedo hacer, sino, ¿qué digo?, veo lo más importante y escribo, no me hace falta" (sujeto 3).

- "Bueno, fácil no es, difícil tampoco, depende un poco... Si me siento que me estoy repitiendo mucho la palabra, lo que es el cambio, un poco de significado de la misma palabra que sea y buscar una serie de sinónimos ¿no? de esa palabra, porque siempre si repito muchas veces la misma palabra pues..., mejor buscar sinónimos" (sujeto 7).

- "Porque hay muchas palabras que no sé lo que significan, entonces pues prefiero quitar esa palabra y pensar otra que sé yo. Para que lo comprendan" (sujeto 10).

De otra parte, hay que señalar que los alumnos sordos, en general, no poseen un suficiente conocimiento del nivel sintáctico del texto, lo cual explica en gran parte las dificultades en el proceso de transcripción. Concretamente, una de las estrategias más conflictivas en la transcripción se refiere a la búsqueda de palabras adecuadas y su estructuración sintáctica, de acuerdo con las reglas gramaticales del lenguaje escrito. A continuación, se exponen algunos resultados más relevantes obtenidos a partir del análisis de los textos escritos por alumnos sordos, clasificados según el nivel macro y microestructural.

Con respecto al nivel oracional-discursivo, se ha encontrado una significativa ausencia de nexos entre proposiciones, que se refiere a cuando éstas están relacionadas semánticamente mediante la coordinación o la subordinación, pero que no aparece la conjunción o el nexo correspondiente que las une. Por ejemplo:

- Ausencia de la conjunción "y" para unir proposiciones que están relacionadas semánticamente por coordinación: “cuando llegó al lago se tumbó () se convertió en princesa" (sujeto 9). 
- Ausencia de la preposición "de" en la oración subordinada sustantiva: “...me doy cuenta () que hay un niño..." (sujeto 2).

- Ausencia del nexo "que" en la oración subordinada adjetiva: "Érase una vez un niño () venía de colegio..." (sujeto 3 ).

- Ausencia de nexos adverbiales en la oración subordinada adverbial: "zorro está susto () viene chico malo" (sujeto 9).

Asimismo, en la mayoría de los textos escritos existe una sustitución de nexos, es decir, un uso de nexos conjuntivos incorrectos. Se trata concretamente del uso de nexos incorrectos en lugar de los correctos. Por ejemplo:

- Sustitución de nexo en la oración coordinada: "El jefe pidió en salvar a su amigo $y$ le negó" (sujeto 6).

- Sustitución de la conjunción "que" por otros nexos en la oración subordinada adjetiva: "Se fueron corriendo y dirigieron a un sitio para coger paracaidas y le salvaron todo excepto el presidente y una ministra para dar mensaje a la Casablanca pidiendo aviones de ejército" (sujeto 6).

- Uso de nexo conjuntivo incorrecto en la oración subordinada adverbial: “...su padre y el señor se fueron a la guerra contra los moros y murió allí, ya que Lazarillo tenía tan sólo ocho años" (sujeto 7).

En relación con el nivel sintagmático-proposicional, se ha observado como la mayoría del alumnado sordo cuando escriben no aplican las reglas de concordancia entre los elementos proposicionales. Por ejemplo, no atienden a la coherencia según el morfema de número que se establece entre el sujeto y el verbo: "Su hermano llamaron a otros cuatro ladrones..." (sujeto 5). Además, al expresar por escrito sus ideas no emplean las formas verbales, constituidas en este caso como núcleo del predicado, como en: "Hola Blancanieve por favor dame agua () sed..." (sujeto 7). Así como también se ha encontrado una ausencia o utilización inapropiada de nexos o preposiciones en el complemento verbal de las proposiciones. Por ejemplo:

- Ausencia del pronombre personal, que desempeña la función de complemento directo e indirecto: "Se acercó a un ruso y () eliminó" (sujeto 3).

- Uso de nexo incorrecto, como en: "entra despacio y a puntillas" (sujeto 3).

\section{Discusión y conclusiones}

Esta investigación se ha centrado en la expresión escrita de alumnos sordos y, en concreto, en el proceso de transcripción. El aprendizaje de la escritura, imprescindible para los adolescentes sordos, se ha revelado especialmente complicado. El estudio sistemático de los procesos cognitivos en la composición escrita de alumnos sordos permite profundizar en sus características específicas, necesarias para la elaboración adecuada de estrategias de enseñanza. De otro lado, el análisis de los resultados del estudio permite realizar algunas reflexiones generales sobre la expresión escrita. 
En resumen, atendiendo a los resultados obtenidos en esta investigación, la mayoría de los alumnos sordos son conscientes de las dudas y las dificultades en la transcripción escrita de sus ideas. En efecto, no poseen las habilidades necesarias para expresar por escrito las ideas de forma adecuada, utilizando correctamente la estructura y la forma del texto.

Hay que señalar que los alumnos sordos, en general, no poseen un suficiente conocimiento del nivel discursivo y sintáctico del texto, lo cual explica en gran parte las dificultades en el proceso de transcripción. Concretamente, una de las estrategias más conflictivas en la transcripción se refiere a la búsqueda de palabras adecuadas, su organización textual y su estructuración sintáctica, de acuerdo con las reglas gramaticales del lenguaje escrito como: la concordancia entre los elementos proposicionales, el orden canónico de los elementos de la oración, la utilización adecuada de verbos, el uso correcto de nexos preposicionales en la estructura del complemento verbal, el empleo apropiado de nexos conjuntivos en las oraciones compuestas,...

La existencia de estas dificultades sintácticas en la expresión escrita de sujetos sordos también se pone de manifiesto en otros estudios, realizados en distintas lenguas (Lacerte, 1989; McAfee, Kelly, y Samar, 1990; Nadeau, Dubuisson y Gélinas-Chebat, 1991; Tur-Kaspa y Dormi, 1998). Varias investigaciones han encontrado que los alumnos sordos poseen un conocimiento insuficiente de las reglas gramaticales de la lengua escrita (Kelly, 1987; Gutiérrez Cáceres, 2004b).

En este sentido, las dificultades en la escritura que experimentan las personas sordas se deben principalmente a las limitaciones lingüísticas y cognitivas, que se producen como consecuencia del déficit experiencial que implica la deficiencia auditiva. Es decir, como consecuencia de la escasa o insuficiente adaptación que el contexto educativo y social realiza según las necesidades y características de las personas sordas.

De otra parte, hay que señalar que las conclusiones acerca del dominio en el proceso de transcripción en la expresión escrita no se pueden generalizar al grupo de alumnos sordos, sobre todo, por la alta variabilidad existente entre los alumnos sordos, en función de las características, necesidades y potencialidades de cada una de ellas.

Partiendo de los resultados de este estudio, las necesidades educativas de los alumnos sordos de Educación Secundaria, en general, relacionadas con la expresión escrita son:

- Integrarse, de manera apropiada y ajustada, en una variedad de experiencias comunicativas, relacionadas con la expresión escrita.

- Percibir, interiorizar, conocer, de forma adecuada y suficiente, las habilidades escritoras relacionadas con el proceso de la transcripción. 
- Utilizar y desarrollar adecuada y funcionalmente las habilidades escritoras relacionadas con el proceso de la transcripción.

- Desarrollar actitudes positivas hacia la escritura, utilizando materiales y procedimientos adecuados a las necesidades comunicativas de los alumnos sordos, con independencia del tipo de lengua.

Para atender estas necesidades educativas se pueden desarrollar diversas actividades prácticas, con el fin de ayudarles a desarrollar la capacidad escritora y, en concreto, mejorar el conocimiento significativo y uso funcional de las habilidades cognitivas, que están relacionadas con el proceso de transcripción de la expresión escrita (véase Salvador Mata, 2000; Salvador Mata y Gutiérrez Cáceres, 2005).

Para ello, es fundamental, ante todo, tener en cuenta la situación concreta de cada alumno, de una parte, y de otra, procurar que exista una adecuada comunicación entre el alumno sordo y las demás personas (familiares, profesores, compañeros del aula,...), con el fin de lograr un aprendizaje participativo y constructivo en la composición escrita de textos narrativos.

Es importante también conocer algunas sugerencias $\mathrm{u}$ orientaciones prácticas generales para diseñar y desarrollar las estrategias educativas según las necesidades de cada alumno, como son:

- Las actividades escritoras deben vincularse a la práctica, o sea, hay que tener en cuenta y partir de los intereses y los conocimientos previos de los alumnos. No tienen que ser nada aburridas y deben tener mucho sentido y ser interesantes para estos niños, trabajando en clase y en casa, por ejemplo, la redacción de una carta para un amigo o un familiar.

- Desarrollar diversas experiencias relacionadas con la escritura, sobre todo, a través de juegos y situaciones donde se desarrolle ante todo una relación de comunicación o una interacción socio-afectiva. De manera que para el alumno sordo realizar las actividades escritoras le resulten amenas y asequibles.

- Utilizar materiales y procedimientos que sean adecuados a las necesidades comunicativas de cada alumno, independientemente del tipo de lengua (lengua oral, lengua de signos,...). De manera que lo importante es que los alumnos tengan un mejor acceso a la información y a los conocimientos.

- El proceso de enseñanza-aprendizaje de la escritura debe realizarse de manera significativa y contextualizada, es decir, no puede realizarse escribiendo repetidamente "mi mamá me mima", sin importar si comprende o entiende lo que está escribiendo. Por ello, es importante plantear preguntas a los alumnos, proponer debates, buscar ejemplos, solucionar las dudas, reflexionar,...

- Para ayudar al alumno a mejorar su escritura, el profesor debe mostrar y explicar claramente el significado y el uso de las estrategias escritoras. No sólo decirle lo que tiene que hacer, sino explicarle por qué y cómo se hace para que lo comprenda. 
- Asimismo, debe explicar las distintas dimensiones del lenguaje escrito como la dimensión discursiva (estructura textual y nexos de cohesión), la dimensión léxicasemántica, la morfo-sintáctica y la fonológica.

- Es importante motivar, despertar o reforzar el interés por la escritura, mostrarles que escribir un texto escrito es una actividad interesante y divertida. Y también es importante que el niño vaya conociendo lo que es la escritura y que vaya viviendo de manera significativa experiencias escritoras para conocer la importancia y funciones de la escritura.

- Es importante también que sea consciente de sus propios errores y que utilice su capacidad de autocorrección (y evalúe el proceso y resultados de su escritura, y de este modo) desarrolla habilidades metacognitivas que hacen posible una mejor expresión escrita. Para ello, es necesario utilizar las guías de escritura y partiendo de preguntas y sugerencias de reflexión.

- Para escribir un texto, hay que centrarse ante todo no en las palabras sino en las unidades significativas del texto, o sea, en el contenido, significado o ideas. Ya que el significado se expresa no en las palabras, sino en las frases, oraciones e incluso párrafos. En este sentido, es importante enseñar a escribir textos y no frases aisladas.

Por último, conscientes de la necesidad de seguir estudiando en profundidad acerca de la expresión escrita en personas sordas, algunas propuestas útiles de investigación son las que se plantean a continuación:

- Contrastar los resultados de esta investigación con otros estudios del proceso de transcripción en personas sordas, realizados con otros instrumentos de obtención y análisis de datos: hablar en voz alta mientras escribe, enfoque ecológico,...

- Establecer relación entre los diversos procesos cognitivos en la expresión escrita de personas sordas: planificación, transcripción y revisión.

- Analizar la conexión entre el dominio de las habilidades relacionadas con el proceso de transcripción y el conocimiento y/o uso de la lengua por parte de las personas sordas (lengua oral/lengua de signos).

Siguiendo esta línea de trabajo y partiendo de la importancia de la escritura ante todo como medio de comunicación y de aprendizaje, es crucial en cualquier caso plantear como objetivo de investigación analizar cómo apoyar a las personas sordas a mejorar la expresión escrita, según sus necesidades y características tanto individuales como contextuales. 


\section{Referencias bibliográficas}

BERNÁRDEZ, E. (Comp.) (1987). Lingüistica del texto. Madrid: Arcos/Libros.

BERNINGER, V.W. \& WHITAKER, D. (1993). Theory Based Branching Diagnosis of Writing Disabilities. School Psychology Review, 22 (4), 623-42.

BERNINGER, V.; WHITAKER, D. \& Otros (1996). Assessment of Planning, Translating, and Revising in Junior High Writers. Journal of School Psychology, 34 (1), 23-52.

BOISCLAIR, A. \& SIROIS, P. (1996). La production de texte chez des élèves malentendants ou sourds. International Journal of Psychology, 31 (3-4), 18436.

CAMBRA, C. (1994). An Instructional Program Approach to Improve Hearing-Impaired Adolescents' Narratives: A Pilot Study. Volta Review, 96 (3), 237-46.

CAMBRA, C. (1993). Proceso de composición de textos narrativos escritos por alumnos sordos. Revista de Logopedia, Foniatria y Audiologia, 13 (2), 73-78.

CARRILlO, M. \& DOMÍNGUEZ, A. B. (Coords.) (2010). Dislexia y sordera: lineas actuales en el estudio de la lengua escrita y sus dificultades. Archidona: Aljibe.

FERNÁNDEZ VIADER, M. P. \& Pertusa, E. (1996). Reflexiones sobre la escritura y la alfabetización de los niños sordos. Revista de Logopedia, Fonología y Audiologia, 16 (2), 79-85.

FERREIRO, E. (1979). ¿Qué está escrito en una oración escrita? Una respuesta evolutiva. Infancia y Aprendizaje, 5, 20-31.

FLOWER, L. \& HAYES, J. R. (1981). A cognitive process theory of writing. College Composition and Communication, 32 (4), 365-387.

FLOWER, L. \& HAYES, J. R. (1984). Images, plans and prose: The representation of meaning in writing. Written Communication, 1 (1), 120-160.

GARCÍA SÁNCHEZ, J. N. (2002). El enfoque de la psicología de la escritura como marco para la evaluación e intervención en la composición escrita. Bordón, 54 (1), 5367.

GUTIÉRREZ CÁCERES, R. (2004a). Estudio descriptivo sobre actitudes hacia la escritura en alumnos sordos de educación secundaria. Educación y Futuro, 1-7.

GUTIÉRREZ CÁCERES, R. (2004b). Cómo escriben los alumnos sordos. Archidona: Aljibe.

GUTIÉRREZ CÁCERES, R. (2005a). Problemas relacionados con la audición: evaluación e intervención educativa. En: Salvador Mata, F. (coord.), Bases Psicopedagógicas de la Educación Especial (pp. 105-126). Archidona: Aljibe.

GUTIÉRREZ CÁCERES, R. (2005b). El proceso de auto-regulación en la expresión escrita de alumnos sordos: estudio de caso en educación secundaria. Revista de Ciencias de la Educación, 203, 403-422. 
GUTIÉRREZ CÁCERES, R. (2005c). La expresión escrita de alumnos sordos. En: Salvador Mata, F. (coord.), La expresión escrita de alumnos con necesidades educativas especiales (pp. 71-87). Archidona: Aljibe.

GUTIÉRREZ CÁCERES, R. (2005d). El proceso de revisión en la expresión escrita de alumnos sordos de Educación Secundaria. Bordón, 57, 647-658.

GUTIÉRREZ CÁCERES, R. Y SALVADOR MATA, F. (2006). El proceso de planificación en la expresión escrita de alumnos sordos: estudio de casos en educación secundaria. Revista de Educación, 339, 435-453.

GUTIÉRREZ CÁCERES, R. Y SALVADOR MATA, F. (2010). Procesos cognitivos y metacognitivos en la expresión escrita de alumnos sordos de Educación Secundaria. En: Carrillo, M. y Domínguez, A. B. (Coords.), Dislexia y sordera: líneas actuales en el estudio de la lengua escrita y sus dificultades (pp. 221-236). Archidona: Aljibe.

GUTIÉRREZ CÁCERES, R. (2002a). Disfunciones sintácticas en los textos escritos por alumnos con deficiencia auditiva de Educación Primaria: análisis descriptivo. Revista de Educación de la Universidad de Granada, 15, 197-210.

GUTIÉRREZ CÁCERES, R. (2002b). Finalista Premio FIAPAS 2002. La expresión escrita de alumnos con deficiencia auditiva: análisis de textos. FIAPAS, 87, 1-12.

HALLIDAY, M. A. K. \& HASAN, R. (1976). Cohesion in English. Londres: Longman.

HAYES, J. R. (1996). A new framework for understanding cognition and affect in writing. En: Levy, C. M. \& Randsell, S. E. (Eds.), The science of writing: theories, methods, individual differences and applications (pp. 1-27). Mahwah: Lawrence Erlbaum.

HOLBROOK, A. P. (1989). Escritura: Enseñanza. En: Husen, T \& Neville, T. (Eds.), Enciclopedia Internacional de la Educación (pp. 2344-2346). Madrid: M.E.C..

KELLY, L. P. (1987). The influence of syntactic anomalies on the writing processes of a deaf collage student. En: Matsuhashi, A. (Ed.), Writing in real time: modelling production processes (pp. 161-196). Norwood, NJ: Ablex.

JÁUDENES, C. (Dir.) (2009). FIAPAS: Estudios sobre población con sordera en España. FIAPAS, 130, Monográfico.

LACERTE, L. (1989). L'ecriture sourde quebecoise. Revue Quebecoise de Linguistique Theorique et Appliquée, 8 (3-4), 303-345.

MCAFEE, M.C.; KELLY, J.F. \& SAMAR, V.J. (1990). Spoken and written english errors of postsecondary students with severe hearing impairment. Journal of Speech and Hearing Disorders, 55 (4), 628-634.

NADEAU, M.; DUBUISSON, C. \& GÉLINAS-CHEBAT, C. (1991). Le Francais ecrit des etudiants sourds: Une Analyse qualitative. Bulletin de l'ACLA, 13 (1), 89-103.

PACE, C.; PONTECORVO, C. \& OTROS (1994). Le prime ipotesi dei bambini sordi sulla lingua scritta. Eta evolutiva, 48, 22-41. 
PERTUSA, E. \& FERNÁNDEZ VIADER, M. P. (1999). Representación fonológica, aprendizaje de la escritura y alumnos sordos. Revista de Logopedia, Foniatría y Audiologia, 19 (1), 2-10.

RAMSPOTT, A. (1991). La comprensión y la producción de cuentos en niños y adolescentes sordos. Tesis Doctoral. Universidad Autónoma de Barcelona.

SALVADOR MATA, F. (1984). Estructuras sintácticas de la lengua escrita en el Ciclo Medio de E.G.B.: análisis evolutivo y diferencial. Tesis doctoral. Madrid: U.N.E.D..

SALVADOR MATA, F. (1997). Dificultades en el aprendizaje de la expresión escrita. Archidona: Aljibe.

SALVADOR MATA, F. (2005). Procesos cognitivos en la expresión escrita: modelos teóricos e investigación empírica. En: Salvador Mata, F. (coord.), La expresión escrita de alumnos con necesidades educativas especiales (pp. 15-44). Archidona: Aljibe.

SALVADOR MATA, F. (Dir.) (1999). El aprendizaje lingüistico y sus dificultades (Sintaxis de la expresión verbal). Granada: Grupo Editorial Universitario.

SALVADOR MATA, F. Y GARCÍA GUZMÁN, A. (2005). Metodología de la investigación. En: Salvador Mata, F. (Coord.), La expresión escrita de alumnos con necesidades educativas especiales. Procesos cognitivos (pp. 45-70). Archidona: Aljibe.

SALVADOR MATA, F. Y GUTIÉRREZ CÁCERES, R. (2005). Atención educativa al alumnado con dificultades en lectura y escritura. Archidona: Aljibe.

SALVADOR MATA, F. (2000). Cómo prevenir las dificultades en la expresión escrita. Archidona: Aljibe.

SCARDAMALIA, M. \& BEREITER, C. (1986). Research on written composition, en C. M. Wittrock (Ed.), Handbook of research on teaching ( $3^{\mathrm{a}}$ ed.) (pp. 778-803). Nueva York: McMillan.

SINCLAIR, H. (1967). Acquisition du langage et dévelopment de la pensée. Paris: Dunlop.

Teruggi, L. A. (2001). Pratiche di lectura e di scrittura nei bambini sordi. Età Evolutiva, 68, 81-86.

TERUGGI, L. A. (2003). Il processo di concettualizzazione della lengua scritta nei bambini sordi. En: Teruggi, L. A. (Ed.), Una scuola, due lingue. L'esperienza di bilingüismo della scuola dell'Infanzia ed Elementare di Cossato (pp. 313-333). Milano: Franco Angeli.

TUR-KASPA, H. \& DROMI, E. (1998). Spoken and written language assessment of orally trained children with hearing loss: Syntactic structure and deviations. Volta Review, 100 (3), 186-202.

VAN DIJK, T. A. (1983). La ciencia del texto. Barcelona: Paidós.

VIGOTSKY, L. S. (1977). Pensamiento y lenguaje. Buenos Aires: La Pléyade. 
VIGOTSKY, L. S. (1978). Mind and society: the development of higher psychological processes. Cambridge: Harvard University Press.

WATSON, L. M. (1999). Literacy and deafness: the challenge continues. Deafness and Education International, 1 (2), 96-107.

ZESIGER, P., DEONNA, T. \& MAYOR, C. (2000). L'acquisition de l'écriture. Enfance, 3, 295-304.

\section{Correspondencia con la autora}

Rafaela Gutiérrez Cáceres

Dpto. Didáctica y Organización Escolar

Facultad Ciencias de la Educación. Universidad de Almería

Ctra. Sacramento, $\mathrm{s} / \mathrm{n}$.

La Cañada de San Urbano (04120, Almería)

E-mail: rcaceres@ual.es 